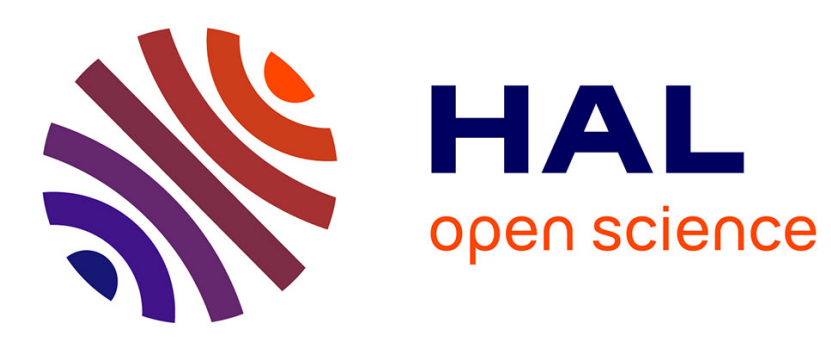

\title{
Des antidotes pour une éthique de l'innovation
}

Léo Coutellec, Paul-Loup Weil-Dubuc

\section{To cite this version:}

Léo Coutellec, Paul-Loup Weil-Dubuc. Des antidotes pour une éthique de l'innovation. Soins, 2019, 64 (837), pp.56-59. 10.1016/j.soin.2019.05.011 . hal-02624244

\section{HAL Id: hal-02624244 \\ https://hal.science/hal-02624244}

Submitted on 26 May 2020

HAL is a multi-disciplinary open access archive for the deposit and dissemination of scientific research documents, whether they are published or not. The documents may come from teaching and research institutions in France or abroad, or from public or private research centers.
L'archive ouverte pluridisciplinaire HAL, est destinée au dépôt et à la diffusion de documents scientifiques de niveau recherche, publiés ou non, émanant des établissements d'enseignement et de recherche français ou étrangers, des laboratoires publics ou privés. 
réflexion

\section{Des antidotes pour une éthique de l'innovation}

LÉo Coutellec* Maître de conférences en éthique et épistémologie des sciences contemporaines, chercheur en éthique, EA1610

PAul-Loup Well-Dubuc Responsable du pôle recherche

Espace éthique île-de-France laboratoire d'excellence Distalz, université Paris-Sud/ Paris-Saclay, bâtiment 300 91405 Orsay cedex, France

*Auteur correspondant Adresse e-mail : leo.coutellec@u-psud.fr (L. Coutellec)



Le champ de la recherche biomédicale sur la maladie d'Alzheimer est particulièrement exposé aux promesses d'innovation.

C 2019 Publié par Elsevier Masson SAS innovation and encourage critical analysis.

() 2019 Published by Elsevier Masson SAS omme tous les domaines à forts enjeux socioéconomiques et scientifiques, le champ de la recherche biomédicale sur la maladie d'Alzheimer est particulièrement exposé aux promesses d'innovation. Dans l'un de ses rapports sur l'économie digitale de 2014, l'Organisation de coopération et de développement économique (OCDE) préconisait d' "accélérer l'innovation dans le traitement de la maladie d'Alzheimer et de la démence » en développant et renforçant "l'incroyable puissance » des big data [1]. Paradigme
II existe une certaine rhétorique messianique dans les discours contemporains sur l'innovation caractérisés par les notions d'attente et de promesse I Depuis la pratique de l'éthique, nous disposons de quelques antidotes pour mettre à distance, voire invalider, la possibilité d'une innovation autoproclamée, et privilégier une réflexion critique.

Mots clés - attente ; éthique ; innovation ; promesse ; rhétorique

Antidotes for ethics in innovation. Contemporary rhetoric regarding innovation is particularly zealous, characterised by notions of expectation and promise. Ethics in care ensure that we now have at our disposal certain antidotes to resist, or even revoke, the possibility of a self-proclaimed

Keywords - ethics; expectation; innovation; promise; rhetoric

scientifique inédit, rupture technologique sans précédent, nouvelle création de valeur, tous les ingrédients sont réunis : "l'innovation innovante va enfin innover". On nous en fait la promesse, chaque année sous de nouveaux habits : dans une décennie pas plus, les maladies neuroévolutives seront enfin sous contrôle technoscientifique. Cependant, de telles promesses, souvent déçues, produisent de la résignation. Alors s'impose une question d'ordre éthique : entre promesse et résignation, autrement dit entre une "espérance sans responsabilité" et une "responsabilité sans espérance" [2], quel espace pouvons-nous ouvrir?

Nous pouvons placer l'idée d'innovation (ici, technoscientifique) face aux biais de ses déterminations idéologiques et proposer quelques antidotes d'une éthique qui chercherait à mettre à distance, voire à invalider, la possibilité d'une innovation autojustificatrice.

\section{UNE RHÉTORIQUE PARTICULIÈRE}

L'innovation semble prise dans le piège de la rhétorique du retard et de l'économie de la promesse.

Dans son enquête historiographique sur le retard, Julie Bouchard, enseignant-chercheur, présente celui-ci comme une "rhétorique de l'insuffisance (constatée) et du changement (attendu) " [3]. Une rhétorique qui se forge philosophiquement, 
selon cette auteure, comme « une abstraction constitutive de la philosophie du progrès " au XVIII ${ }^{\mathrm{e}}$ siècle. Elle rappelle que Anne-Robert-Jacques Turgot use abondamment de la métaphore de la marche dans son Tableau philosophique des progrès de l'esprit humain (1750), là où s'invente "un récit qui ordonne littéralement les peuples en devanciers et en suiveurs » [4]. Elle rappelle aussi cette prophétie de Claude Bernard en 1877 : "Je ne peux m'adresser qu'aux jeunes, qui n'ont pas de parti pris d'avance. Je leur montre la voie nouvelle et je leur dis : suivez-la, car sans cela vous serez en retard. [...] Cette médecine expérimentale, dont je vous parle, arrivera malgrévous, malgré moi, malgré tous ceux qui ne veulent pas en entendre parler, parce qu'elle est le résultat nécessaire et fatal de l'évolution même de la science » [4] là où la rhétorique du retard s'associe au déterminisme scientifique et technique.

I Au xx siècle, cette rhétorique du retard prend de l'ampleur et devient un élément central de l'économie et du comparatisme géographique pour, progressivement, servir de trame idéologique et sémantique au « régime de la promesse technoscientifique» [5].

I Dans son analyse des discours sur le retard dans la période 1940-1970, notamment dans les processus de planification d'après-guerre, Julie Bouchard pointe le rôle majeur joué par la « production des attentes» en matière d'investissement, de recherche et de développement. Pour éviter les prétendus retards, il faut susciter les attentes du public, il faut le faire rêver, créer des futurs désirables, ouvrir les imaginaires technoscientifiques par la promotion d'innovations, si possible "de rupture". Dans un contexte de compétition économique accrue et d'une société de croissance, s'invente alors une véritable économie ou marché de la promesse, dont le discours sur les innovations devient l'otage [6].

I Aujourd'hui, les visions sur le futur se vendent, s'achètent, s'échangent. Le ton prophétique et publicitaire du rapport Converging Technologies for Improving Human Performance (2003) de la National Science Foundation (États-Unis) fut en cela assez paradigmatique. L'exemple plus récent de la façon dont le Human Brain Project a "gagné" un financement européen très important en est un autre [7]. La promesse ici était de simuler le fonctionnement du cerveau avec un supercalculateur, par le développement d'innovations informatiques, inspirées du fonctionnement neuronal et à même de permettre la mise en convergence de données et de connaissances neuroscientifiques, pour développer des stratégies de traitement de maladies dites du cerveau (par exemple, Alzheimer). L'existence et la possibilité de maîtrise d'un "code neural" furent vivement critiquées, y compris par une partie de la communauté neuroscientifique : que peut produire une telle "prophétie épistémopolitique", sinon de l'aveuglement et de la déception?

I Comme dans de nombreux autres cas [8], la promesse et l'attente sont ici coproduites et s'entretiennent mutuellement. L'enjeu éthique se dessine alors : comment sortir les pratiques et politiques d'innovation de cette coproduction qui les enferment dans un mécanisme de prophéties autoréalisatrices par lequel "l'innovation" triomphe avant même d'avoir été évaluée ? Peut-on penser l'innovation sans la rhétorique du retard et l'économie de la promesse? Ces questions peuvent se résumer en une seule, plus fondamentale : peut-on sauver l'idée d'un progrès humain sans céder au messianisme de l'innovation technique?

\section{L'AMBIGUIITÉ DU CONCEPT D'INNOVATION}

I Le premier travail à mener est sémantique. En lui-même, le mot "innovation" est porteur d'une ambiguïté. Le qualificatif "innovant" décrit un objet comme nouveau autant qu'il en loue les qualités. Il est tout à la fois descriptif et évaluatif et pourrait ainsi s'apparenter à ce que les philosophes Hilary Putnam et Bernard Williams appellent des «concepts éthiques épais » [9]. L'innovation est (nouvelle) et doit être (meilleure). Le qualificatif de "responsable", que l'on semble vouloir lui associer désormais systématiquement, ne fait que renforcer cette caractéristique. De cette ambiguité jouent les producteurs d'innovations pour opposer aux sceptiques une double critique implicite : douter qu'un produit ou un dispositif est "innovant" revient à douter d'un fait évident (sa nouveauté) ; mais ce serait aussi, dans le même temps, s'opposer à ce qui est nouveau par la valorisation conformiste de l'existant. Piège idéologique qui nous fait comprendre pourquoi ce concept, véritable "mot d'ordre" qui invite à "tout changer pour ne rien changer » [10], est devenu le motif le plus sollicité du marché de la promesse.

I Faut-il alors se résigner à abandonner le mot lui-même pour éviter les pièges qu'il charrie et revenir à des concepts plus classiques ? Cependant, les risques associés à l'usage du 


\section{dossier}

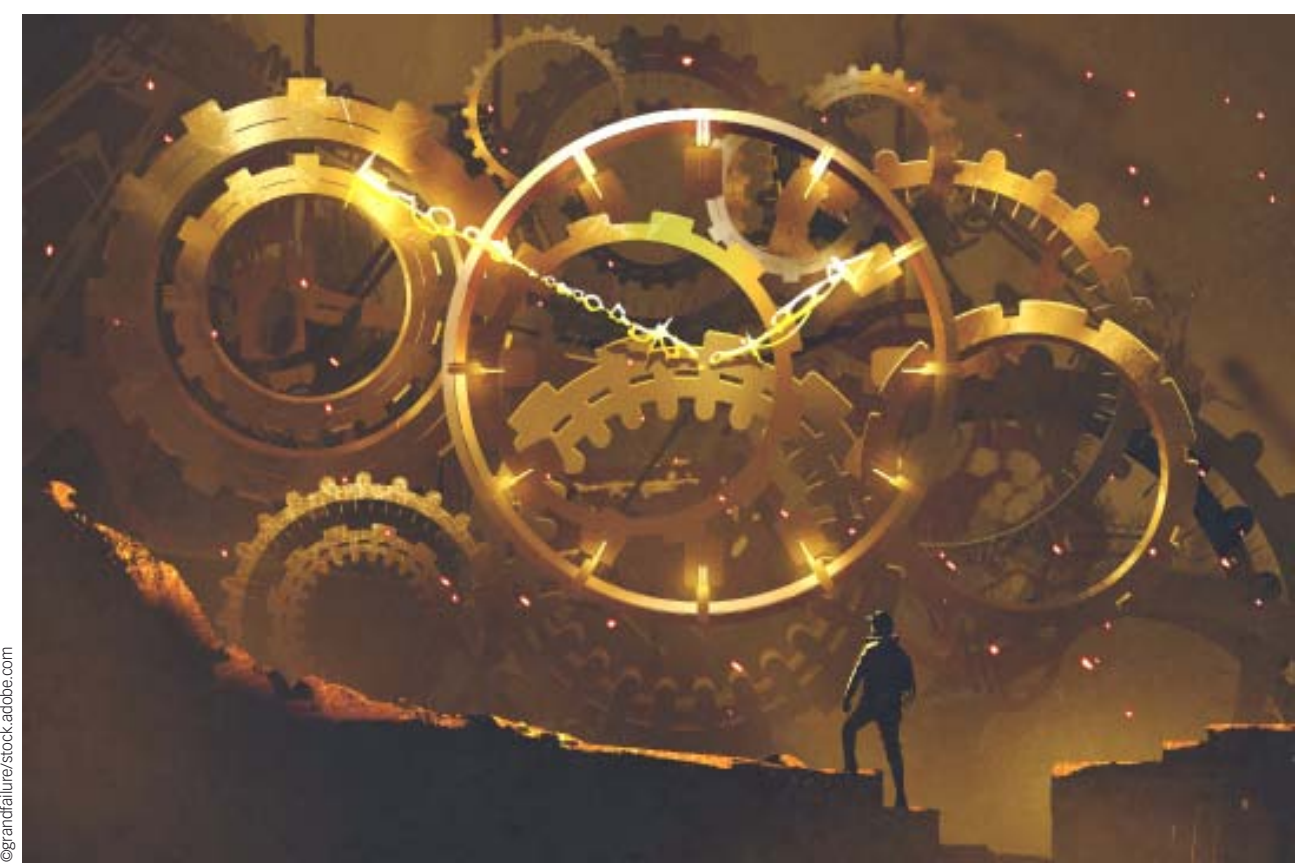

L'innovation semble prise dans le piège de la rhétorique du retard et de l'économie de la promesse.

terme progrès technique, c'est-à-dire d'un progrès inéluctable et linéaire réduit à sa dimension technique, ne sont-ils pas du même ordre?

\section{RéFÉRENCES}

[1] Organisation de coopération et de développement économiques (OECD). Unleashing the Powe of Big Data for Alzheimer's Disease and Dementia Research. Main Points of the OECD Expert Consultation on Unlocking Global Collaboration to Accelerate Innovation for Alzheimer's Disease and Dementia. OECD Digital Economy Papers. 2014;(233):1-40. [2] Coutellec L, Moser JS, Hirsch E, Weil-Dubuc PL. Towards an Ethics of Anticipation, between Hope and Responsibility: French Perspectives in the Case of Alzheimer's Disease. In: Dubljević V. Living with Dementia: Neuroethical Issues in International Perspectives. Basingstoke (Royaume-Uni): Springer Nature; 2019 [3] Bouchard J. Comment le retard vient aux Français. Analyse d'un discours sur la recherche, l'innovation et la compétitivité 1940-1970. Villeneuve-d'Ascq: Presses universitaires du Septentrion; 2008.

Ancrée sur l'analyse fine et concrète des objets techniques et de leur milieu, nous pouvons formuler la praxis d'une éthique de l'innovation comme la production d'antidotes à l'innovation autojustificatrice.

I Un premier antidote consiste à penser les conditions d'une évaluation de la pertinence des innovations. L'analyse simondonienne des objets techniques offre une piste intéressante : l'objet est ce dont il est la genèse et il crée son propre milieu [11]. Il s'agit donc d'orienter notre attention éthique vers les processus d'individuation des objets techniques, leur mode d'existence et l'étude du milieu technique ainsi créé. Évaluer le caractère innovant d'un dispositif technique impliquerait alors bien plus qu'une inscription dans un catalogue de nouveautés. Il s'agirait plutôt de discuter des conséquences sociales de son fonctionnement, des réponses adaptatives du milieu, des déterminations sociotechniques qu'il entraîne, des soubassements épistémologiques qui le forment. Des réponses qui ne sont pas toujours prévisibles. Il se pourrait ainsi que la rencontre entre les caractères "innovant" et "pertinent" d'un objet technique ne se produise que bien des années après sa mise en place, voire jamais. Ainsi, l'évaluation du milieu technique (dans toutes ses dimensions), et non plus seulement celle de l'objet technique, devient un enjeu éthique.
Un deuxième antidote vise à déconstruire l'assertion selon laquelle "pour faire avancer la connaissance, il n'y a qu'à essayer ", qui se traduit dans sa "forme forte" par : "Tout ce qui peut être essayé sera tenté », selon les mots de Nicolas Bouleau [12]. Celui-ci qualifie cette idée de faute de raisonnement car cela revient "à croire que le futur peut passer par tous les possibles, ce qui est absurde » [12]. Il s'agit donc de défendre l'idée selon laquelle l'ordre des avancées scientifiques 
et techniques (c'est-à-dire ce que l'on considère comme pertinent ou prioritaire de faire en science et innovation) doit être l'objet d'une discussion très ouverte. Cet aspect est magnifiquement illustré dans la phrase suivante : "[...] l'horizon s'est embrumé, le bateau arrive dans une zone de récifs, la joie de naviguer est importante mais ne suffit pas, ily a lieu de choisir la direction de ses efforts. » [12] Parce que tout n'est pas possible et que tout ne doit pas être possible, cette forme de réalisme prudent appelle effectivement à choisir la direction de ses efforts pour comprendre, par exemple, que "ré-orienter l'économie vers le respect de la biosphère de sorte que cette réorientation soit une dynamique autoréalisatrice " est beaucoup plus difficile que «d'insérer tel ou tel segment de bases dans un ADN pour voir ce que ça fait » [12].

I Un troisième antidote viserait à prendre en compte sérieusement les peurs et les craintes. Ici, il s'agit de sortir du régime de la promesse pour partager des craintes. Ce sont une science et une technique moins rassurantes qui se dessinent, moins positives, une technoscience qui « perd la vertu de fournir plus de sécurité » mais "gagne par la possibilité de fournir plus de lucidité» [12]. Il ne s'agit pas de substituer au marché de la promesse un marché de la catastrophe mais de changer l'horizon d'attente en faisant des craintes des préoccupations à traiter collectivement qui renforcent nos capacités « à interpréter les indices de difficultés à venir » [12]. Abandonner donc définitivement l'idée d'une solution miracle pour cultiver le pluralisme et la «multiplication de l'imparfait » [13].

I Enfin, un quatrième antidote consisterait à associer à cette éthique de l'innovation une réflexion sur les limites de et dans l'innovation. Ici, l'enjeu serait de passer chaque processus d'innovation, ou plus précisément tel objet ou dispositif technique considéré comme innovant, au crible des trois conceptions de la

\section{Les points à retenir}

- Les discours contemporains sur l'innovation sont pris dans une rhétorique du retard et de la promesse.

- Ces promesses sont souvent déceptives et poussent à la résignation.

- Le concept d'innovation lui-même, à la fois descriptif et évaluatif, renforce ce messianisme.

- Il est possible de penser une éthique de l'innovation sans les promesses et les attentes qui lui sont associées. limite : les seuils, les bornes et les frontières [14]. Quels types de seuils se négocient? Quels types de bornes s'imposent? Quels types de frontières s'inventent? Ainsi, parler des limites de l'innovation sera tout autre chose que de choisir entre les partisans de la "pente glissante" et ceux du "train que l'on n'arrête pas". Penser les limites en contexte de production d'innovation technique revient à s'extraire d'une conception bornée de la limite qui ne laisse le choix qu'à deux options, du type "stop ou encore", ignorant ainsi le caractère politique et autobiographique de tout récit sur les limites. Considérant les prétentions d'innovation dans le champ de la maladie d'Alzheimer, par exemple le développement de tests génétiques par le séquençage très haut débit du génome, les questions à se poser pourraient être les suivantes : quel type de seuil doit-on négocier avec de tels tests (par exemple, la question du degré de précocité dans la maladie sur lequel s'appuyer pour faire ces tests) ? Existe-t-il des bornes qui s'imposent à eux (par exemple, l'interdiction du test génétique dans une situation asymptomatique) ? Quelles frontières voulons-nous inventer à leur propos (par exemple, l'émergence de la figure du "malade potentiel", modifiant les frontières classiques entre le normal et la pathologique) ?

\section{Conclusion}

Au-delà des questionnements éthiques légitimes sur le bien-fondé de telle ou telle innovation, notre attention éthique doit se porter sur le cadre de pensée, les concepts et les rhétoriques dans lesquelles ils sont pris. Le discours contemporain sur l'innovation, autant que le concept lui-même, laisse penser qu'au-delà des innovations autoproclamées, il n'est point de salut ; ou encore que toute réflexion critique relèverait du conservatisme. Il nous semble avoir ici montré que, bien au contraire, les stratégies de l'économie de la promesse, ici brièvement analysées, sont empreintes d'une tonalité tout à la fois conservatrice et paternaliste. N'est-ce pas aux enfants à qui l'on promet de satisfaire une espérance préalablement construite?

\section{RÉFÉRENCES}

[4] Bouchard J.L'invention du retard comme rhétorique de l'insuffisance et du changement. Mots. Les langages du politique. 2006;80. https:// journals.openedition.org/mots/564 [5] Joly PB. Le régime des promesses technoscientifiques. In: Audétat M. Sciences et technologies émergentes. Pourquoi tant de promesses? Paris: Hermann; 2015. p. 31-48 [6] Nordmann A. L'espoir technologique pour la planète bleue. In: Grosclaude JY, Pachauri RK, Tubiana L. Regards sur la Terre. Les promesses de l'innovation durable. Paris: Armand Colin; 2014. p. 169-76. [7] Panese F. Cerveau et imaginaire sociotechnique: le Human Brain Project entre science et politique. In:Audétat M. Sciences et technologies émergentes. Pourquoi tant de promesses ? Paris: Hermann; 2015. p. 165-93. [8] Audétat M. Sciences et technologies émergentes. Pourquoi tant de promesses? Paris: Hermann; 2015.

[9] Putnam H. Fait/valeur : la fin d'un dogme et autres essais. Paris: L'Éclat; 2004.

[10] Bontems VK. What does Innovation stand for? Review of a watchword in research policies. Journal of Innovation Economics \& Management. 2014;(15):39-57.

[11] Simondon G. Du mode d'existence des objets techniques. Paris: Aubier; 2012. [12] Bouleau N. Penser l'éventuel. Faire entrer les craintes dans le travail scientifique. Versailles: Quæ; 2017.

[13] Coutellec L, Pintureau B. Crop protection in sciences, ethics and societies. From quick-fix ideal to multiple partial solutions. J Agric Environ Ethics. 2013;26(1):207-30.

[14] Benasayag M, Coutellec L. Nos limites ne sont pas les leurs. De la nécessité d'une approche critique de la notion de limite. Écologie \& politique. 2018;(57):117-32.

Déclaration de liens d'intérêts Les auteurs déclarent ne pas avoir de liens d'intérêts. 